\title{
DESIGUALDADE DE RENDIMENTOS POR GÊNERO INTRA-OCUPAÇÕES NO BRASIL, EM 2004*
}

\author{
Jacqueline Nogueira Cambota ${ }^{* *}$
}

\author{
Paulo Araújo Pontes ${ }^{* * *}$
}

\begin{abstract}
RESUMO Uma das características mais marcantes do mercado de trabalho brasileiro é o diferencial de rendimentos entre raças e gênero. Diante disso, este trabalho pretende verificar se há nesse mercado desigualdade de rendimentos por gênero para os indivíduos alocados em uma mesma ocupação, segundo sua auto-identificação racial. Para tanto, serão utilizados os dados da PNAD 2004 e uma metodologia semiparamétrica - estimador do núcleo. Os exercícios contrafactuais sugerem evidências de discriminação contra mulher intra-ocupação, sendo esta visualmente mais evidente nas ocupações com maior escolaridade. Verificou-se ainda que a desigualdade de rendimentos é maior para os quantis mais elevados da distribuição.
\end{abstract}

Palavras-chave: desigualdade de rendimentos por gênero e discriminação

Código JEL: J31, J71

INEQUALITY OF INCOMES FOR GENDER IN BRAZIL, 2004

ABSTRACT One of main characteristics of Brazilian labor market is the male-female and the black-white wage differential. So this paper looks for evidences of female wage inequality for black and white individuals who are allocated in same occupation. For this aim, we used the data on the PNAD 2004 and a semiparametric method - kernel estimator. Counterfactual simulations showed visually the presence the discrimination against women workers for both races, it was visually higher in the occupation with higher schollarship. The female wage inequality also increased for higher wages.

Key words: female wage inequality and discrimination

\footnotetext{
* Artigo recebido em 28 de setembro de 2006 e aprovado em 13 de março de 2007.

** Professora das Faculdades Cearenses, e-mail: jqnc@click21.com.br

*** Professor das Faculdades Cearenses e Analista de Políticas Públicas do IPECE, e-mail: paulopontes @ipece.ce.gov.bre pauloarpo@click21.com.br
} 


\section{INTRODUÇÃO}

Uma das características mais marcantes do mercado de trabalho brasileiro é o diferencial salarial entre gêneros e raças, sendo a discriminação um dos componentes relevantes na explicação desse diferencial. Ela pode ocorrer de duas formas: por meio da discriminação salarial pura ou da segregação ocupacional. Diz-se que há discriminação pura quando indivíduos igualmente produtivos alocados em posto de trabalho da mesma produtividade recebem rendimentos distintos. Já a segregação ocupacional ocorre quando trabalhadores igualmente produtivos têm chances diferentes de ocupar empregos de maior remuneração.

O Relatório Global sobre Discriminação no Mercado de Trabalho (2003), elaborado pela OIT, expressa que no Brasil homens e mulheres tendem a trabalhar em setores diferentes da economia. As mulheres, normalmente, são empregadas em uma gama mais limitada de ocupações e, comumente, possuem menor jornada de trabalho, sendo esta uma das razões dos menores rendimentos recebidos por elas. Essa segregação ocupacional aparece também em novas áreas de trabalho relacionadas às tecnologias e à comunicação, o que indica a persistência e reprodução da segregação ocupacional por gênero.

A diferença entre gêneros no mercado de trabalho não está somente na inserção; homens e mulheres também recebem rendimentos distintos quando estão alocados em uma mesma ocupação [ver Araújo e Ribeiro (2002), Baptista (2000) e Giuberti e Menezes-Filho (2005)]. Barros et al. (1997) estimam que se o diferencial de rendimento por gênero intra-ocupação não existisse, o diferencial de rendimento por gênero poderia ser reduzido em um terço. Por outro lado, Leme e Wajnman (2000) mostram que, entre os trabalhadores sem carteira e por conta própria, as mulheres estão em maior proporção nas ocupações que contribuem positivamente para seus rendimentos, ou seja, tendem a se dirigir para os setores em que são mais bem remuneradas.

Além dos autores supracitados, outros buscam abordar as desigualdades de gênero concomitantemente com as de raça. De acordo com Soares (2000), o mercado de trabalho brasileiro discrimina homens negros e mulheres brancas e negras, mas a pior situação é a da mulher negra, que sofre com 
uma discriminação setorial-regional-ocupacional maior do que os homens da mesma cor e as mulheres brancas. Cavalieri e Fernandes (1998) verificaram que os homens recebem rendimentos em média 60\% superiores aos das mulheres, sendo essa diferença maior entre os não-brancos do que entre os brancos.

Diante do exposto, este trabalho tem como objetivo identificar e analisar a desigualdade de rendimentos por gênero para indivíduos alocados em grupos ocupacionais idênticos, segundo a auto-identificação racial. A identificação das desigualdades será feita com base em exercícios contrafactuais, que permitiram visualizar se a despeito do mesmo nível de produtividade e da mesma alocação ocupacional ainda persiste o diferencial de rendimentos entre gêneros para indivíduos da mesma raça.

Para tanto, utilizar-se-á uma metodologia semiparamétrica, que é uma adaptação feita por Dinardo et al. (1996) para o estimador do núcleo desenvolvido por Rosenblatt (1956) e Parzen (1962). A principal vantagem desse método é que os resultados são baseados em toda a distribuição de rendimentos, portanto, será possível identificar se a desigualdade aumenta à medida que as mulheres avançam para faixas de rendimentos mais elevados dentro da ocupação.

$\mathrm{O}$ artigo encontra-se dividido em cinco seções, incluindo esta introdução. Na segunda, será feita uma análise exploratória dos dados. Na terceira, far-se-á uma exposição da metodologia utilizada. Nas seções seguintes, serão apresentados os principais resultados, bem como as considerações finais.

\section{BASE DE DADOS}

A base de dados utilizada é extraída da Pesquisa Nacional de Amostra por Domicílio (PNAD) para o ano 2004. A amostra se refere a indivíduos com rendimento positivo no trabalho principal, idade entre 25 e 50 anos, e que se classificaram como brancos, pretos ou pardos. Foram excluídos da amostra indivíduos que trabalhavam por conta própria ou que eram empregadores, pois nesses casos não é possível identificar práticas discriminatórias. Também foram excluídos os indivíduos sem declaração de renda e de horas trabalhadas na semana de referência. 
Após a aplicação desses filtros, a amostra total possui 32.398.703 observações, sendo 9.165.259 homens brancos, 8.467.540 homens negros, 8.421.705 mulheres brancas e 6.344.199 mulheres negras.

\subsection{Produtividade, desigualdade de rendimentos intra-ocupação e distribuição ocupacional}

Segundo Ramos e Vieira (2001), as diferenças salariais podem ser provenientes das diferenças nos atributos produtivos dos trabalhadores, entre os quais, vale destacar, por normalmente se revelarem de maior importância, a educação e a experiência.

A relação entre educação e desigualdade salarial depende do grau de desigualdade entre os trabalhadores e da sensibilidade dos trabalhadores ao nível educacional. No Brasil, a influência da educação para a desigualdade salarial é muito elevada, pois, além de ser um país com um dos maiores graus de desigualdade em educação, também é um dos países com a maior sensibilidade dos salários em relação ao nível educacional.

Lam e Levison (1990) estimam que o grau de desigualdade educacional no Brasil é cerca de seis vezes superior ao observado nos Estados Unidos. O impacto desse elevado grau de desigualdade sobre os salários depende da sensibilidade do mercado de trabalho ao nível educacional, visto que quanto maior (menor) a importância da educação para o mercado de trabalho maior (menor) será seu impacto sobre a desigualdade.

Nesse sentido, são apresentados na tabela 1 dados sobre educação por gênero, segundo a raça, para os oito grupos ocupacionais estudados. Nesta

Tabela 1: Anos de estudo médios por gênero, segundo a raça e o grupo ocupacional, 2004

\begin{tabular}{|c|c|c|c|c|}
\hline \multirow[b]{2}{*}{ Grupos ocupacionais do trabalho principal } & \multicolumn{2}{|c|}{ Brancos } & \multicolumn{2}{|c|}{ Negros $^{1}$} \\
\hline & Feminino & Masculino & Feminino & Masculino \\
\hline Dirigentes em geral & 14,05 & 12,71 & 13,12 & 10,98 \\
\hline Profissionais das ciências e das artes & 15,31 & 15,04 & 14,79 & 14,12 \\
\hline Técnicos de nível médio & 12,90 & 12,41 & 12,19 & 11,14 \\
\hline Trabalhadores administrativos & 12,41 & 11,55 & 11,79 & 10,57 \\
\hline Trabalhadores dos serviços & 7,22 & 8,10 & 6,67 & 7,24 \\
\hline Trabalhadores do comércio & 10,75 & 9,97 & 10,00 & 8,60 \\
\hline Trabalhadores agrícolas & 5,02 & 4,65 & 4,20 & 3,55 \\
\hline Trab. produção de bens e serviços e manutenção & 8,40 & 8,04 & 8,12 & 7,02 \\
\hline
\end{tabular}


tabela observa-se que, com exceção dos trabalhadores do serviço, as mulheres têm em média um ano de estudo a mais do que os homens para ambas as raças. Verifica-se, no entanto, que dentro da raça negra a diferença de escolaridade por gênero é maior. Contata-se, ainda na tabela 1, que o perfil de escolaridade no interior da raça branca é mais elevado do que o dos indivíduos da raça negra.

Já a relação entre experiência e produtividade pode ser mensurada por meio de dois tipos de experiência: a no mercado de trabalho (tempo que um indivíduo trabalha) e a no emprego atual (tempo que o trabalhador está no emprego atual). Esta última busca medir a rotatividade da mão-de-obra.

Uma das dificuldades do estudo da relação entre experiência no mercado de trabalho e desigualdade salarial advém do fato de não dispormos de informações diretas sobre a experiência de um trabalhador. Diante disso, calcularam-se as idades médias dos trabalhadores como proxy para a experiência no mercado de trabalho, expostas na tabela 2. Nesta tabela, é possível observar uma baixa diferença de idade entre gêneros, o que sugere que, independente da raça e da ocupação, a experiência de homens e mulheres é muito próxima. No entanto, como as mulheres possuem maior idade média, sua experiência é um pouco maior do que a dos homens.

Portanto, os dados na página seguinte mostram que existe um diferencial de produtividade a favor das mulheres; no entanto, este não se reflete em maiores prêmios salariais para elas. Esta última constatação pode ser observada a partir da análise da tabela 3, na qual são apresentadas as médias de rendimento-hora segundo o gênero, raça e grupo ocupacional, em que é

Tabela 2: Idade média por gênero, segundo a raça e o grupo ocupacional, 2004

\begin{tabular}{lcccc}
\hline & \multicolumn{2}{c}{ Brancos } & \multicolumn{2}{c}{ Negros } \\
\hline Grupos ocupacionais do trabalho principal & Feminino & Masculino & Feminino & Masculino \\
\hline Dirigentes em geral & 36,81 & 37,77 & 36,55 & 36,75 \\
\hline Profissionais das ciências e das artes & 36,62 & 36,51 & 36,37 & 35,95 \\
\hline Técnicos de nível médio & 36,30 & 35,88 & 35,50 & 35,47 \\
\hline Trabalhadores administrativos & 34,89 & 35,29 & 34,66 & 34,93 \\
\hline Trabalhadores dos serviços & 37,51 & 36,29 & 36,74 & 35,48 \\
\hline Trabalhadores do comércio & 33,56 & 33,78 & 32,78 & 33,72 \\
\hline Trabalhadores agrícolas & 35,76 & 36,48 & 36,49 & 35,71 \\
\hline Trabalhadores da produção de & 35,78 & 35,97 & 35,13 & 35,58 \\
bens e serviços e manutenção & & & & \\
\hline
\end{tabular}


Tabela 3: Rendimento-hora médio, segundo a raça e o tipo de ocupação, 2004

\begin{tabular}{lrrrrrrr}
\hline Grupos ocupacionais do & \multicolumn{4}{c}{ Branco } & \multicolumn{4}{c}{ Negro } \\
\cline { 2 - 3 } trabalho principal & 11,36 & 15,16 & $-25,09$ & 10,06 & 10,75 & $-6,44$ \\
\hline Dirigentes em geral & 6,79 & 9,50 & $-28,53$ & 4,83 & 6,28 & $-23,16$ \\
\hline Profissionais das ciências e das artes & 11,03 & 20,42 & $-45,97$ & 8,34 & 11,95 & $-30,21$ \\
\hline Técnicos de nível médio & 4,92 & 6,50 & $-24,32$ & 4,14 & 4,34 & $-4,59$ \\
\hline Trabalhadores administrativos & 2,50 & 3,50 & $-28,73$ & 2,08 & 2,85 & $-27,12$ \\
\hline Trabalhadores dos serviços & 3,37 & 4,42 & $-23,73$ & 2,41 & 3,05 & $-20,77$ \\
\hline Trabalhadores do comércio & 1,59 & 1,95 & $-18,36$ & 1,29 & 1,63 & $-20,96$ \\
\hline Trabalhadores agrícolas & 2,77 & 4,00 & $-30,83$ & 2,16 & 3,22 & $-32,86$ \\
\hline Trabalhadores da produção de & & & & & & \\
bens e serviços e manutenção & & & & &
\end{tabular}

Fonte: Elaboração própria a partir dos dados da PNAD 2004.

Tabela 4: Coeficiente da razão entre trabalhadores mulheres

e homens conforme a raça segundo o tipo de ocupação, 2004

\begin{tabular}{lcc}
\hline Grupos ocupacionais do trabalho principal & Branco & Negro \\
\hline Dirigentes em geral & 0,65 & 0,50 \\
\hline Profissionais das ciências e das artes & 1,86 & 1,84 \\
\hline Técnicos de nível médio & 1,05 & 1,32 \\
\hline Trabalhadores administrativos & 1,63 & 1,29 \\
\hline Trabalhadores dos serviços & 2,31 & 2,18 \\
\hline Trabalhadores do comércio & 1,04 & 0,71 \\
\hline Trabalhadores agrícolas & 0,17 & 0,14 \\
\hline Trabalhadores da produção de bens e serviços e manutenção & 0,21 & 0,13 \\
\hline
\end{tabular}

Fonte: Elaboração própria a partir dos dados da PNAD 2004.

possível constatar a maior média salarial dos homens em todos os casos. Com exceção dos trabalhadores ocupados no serviço, na agricultura e na produção, o diferencial de rendimentos entre gêneros é maior dentro da raça branca, alcançando uma diferença de quase $50 \%$ na ocupação de profissionais das ciências e das artes.

O coeficiente ${ }^{2}$ da razão entre trabalhadores mulheres e homens mostra que existe no mercado de trabalho brasileiro uma divisão clara entre ocupação feminina e masculina. Os trabalhadores do sexo masculino são maioria nos seguintes grupos ocupacionais: dirigentes em geral; trabalhadores agrícolas; e trabalhadores na produção de bens e serviços de manutenção. Já os grupos ocupacionais com predominância de trabalhadoras do sexo feminino são os seguintes: profissionais das ciências e das artes; técnicos de nível médio; trabalhadores administrativos; e trabalhadores dos serviços. Apenas 
a ocupação de trabalhadores do comércio variou entre feminina e masculina de acordo com a raça.

Observa-se, então, que, embora três das ocupações tipicamente femininas estejam entre as de maior rendimento (profissionais das ciências e das artes, técnicos de nível médio e trabalhadores administrativos), a maior presença das mulheres nessas ocupações não foi suficiente para alcançar uma menor desigualdade de rendimentos (tabela 3). Portanto, as mulheres têm em média maior nível de produtividade, recebem rendimentos menores e são maioria nas ocupações de maior rendimento. Essas estatísticas revelam evidências de que mais grave que a segregação ocupacional é a diferença de rendimentos por gênero intragrupos ocupacionais.

\section{METODOLOGIA}

Nesta seção será apresentada a metodologia não paramétrica para a estimação de funções densidades — estimador do núcleo, bem como a adaptação deste feita por Dinardo et al. (1996) para a estimação de densidades contrafactuais.

\subsection{Estimador do núcleo e estimador do núcleo ponderado}

O estimador do núcleo $\hat{f}(x)$ de uma densidade univariada $f(X)$, baseada em uma amostra aleatória $X_{1}, X_{2}, X_{3}, \ldots ., X_{n}$ de tamanho $n$, é definido, de acordo com Rosenblatt (1956) e Parzen (1962), como:

$$
\hat{f}(x)=\frac{1}{n h} \sum_{i=1}^{n} K\left(\frac{x-X i}{h}\right)
$$

Sendo:

$K(\cdot)$ : é uma função simétrica chamada núcleo, que dá nome ao estimador, satisfazendo as seguintes propriedades:

$$
\int_{-\infty}^{\infty} K(t) d t=1, \quad \int_{-\infty}^{\infty} t K(t) d t=0 \quad \text { e } \quad \int_{-\infty}^{\infty} t^{2} K(t) d t=k \neq 0 .
$$

Quando $K(\cdot)$ for uma função não negativa, ela será uma função densidade de probabilidade, o que implica que $\hat{f}(x)$ será também uma função densidade de probabilidade. 
$h$ : é a largura dos intervalos de classes (bandwidth), também conhecida como parâmetro de suavização.

Assim sendo, a função densidade estimada, $\hat{f}(x)$, dependerá do formato da função núcleo e do parâmetro de suavização $h$. Em geral, o critério para a determinação ótima de $h$ é minimizar o erro quadrático médio integrado (EQMI), definido como:

$$
\begin{aligned}
& \underset{\operatorname{EQMI}}{\operatorname{E}(\hat{f}(x))=\int E\{\hat{f}(x)-f(x)\}^{2} d x=\int\{E[\hat{f}(x)]-f(x)\}^{2} d x+\int} \\
& \operatorname{var} \hat{f}(x) d x
\end{aligned}
$$

Como a eficiência dos diversos núcleos em minimizar o erro quadrado médio não varia muito, optou-se pelo núcleo gaussiano, já que a distribuição da variável logaritmo neperiano do rendimento por hora se mostrou aproximadamente com distribuição normal. Para esse núcleo, Silverman (1986) mostra que o parâmetro de suavização ótimo, $h_{o t m}$, é dado por $h_{o t m}=$ $1,06 \sigma n^{-1,5}$, sendo, $\sigma$ o desvio-padrão da distribuição gaussiana. Uma estimativa robusta de $\sigma$ é dada por $A=\min$ (desvio-padrão, desvio interquartil/1,34). Nesses termos, o parâmetro de suavização ótimo utilizado será:

$$
h_{\text {otm }}=0,9 A n^{-1,5}
$$

Já para o cálculo das densidades contrafactuais usou-se a adaptação feita por Dinardo et al. (1996) para o estimador do núcleo. Esse método deriva da conhecida decomposição de Oaxaca (1973), que se baseia em exercícios contrafactuais. Tais exercícios buscam tornar homens e mulheres idênticos produtivamente, pois, ao atribuir as características produtivas dos homens às mulheres, tem-se que a distância entre suas densidades deve-se a uma possível presença da discriminação no mercado de trabalho, dada uma mesma alocação ocupacional.

Desse modo, o cálculo das densidades contrafactuais é feito considerando cada observação individual como um vetor $(w, p, g)$, em que $w$ é o logaritmo neperiano de rendimento/hora, $p$ é o atributo produtividade, que será medido em termos de educação e idade, e $g$ é o gênero do indivíduo. Os atributos produtivos se dividem em cinco células de educação e cinco de idade, perfazendo um total de 25 células para cada ocupação, segmenta- 
das de acordo com o gênero e a auto-identificação racial. A distribuição conjunta de $(w, p, g)$ será representada por $F(w, p, g)$. A distribuição conjunta de rendimentos $(w)$ e atributo $(p)$, dado o gênero $(g)$, será representada por uma distribuição condicionada $F(w, p / g)$.

Assim, por exemplo, a densidade de rendimentos das mulheres, $f_{m}(w)$, pode ser escrita como a integral de suas densidades condicionada ao seu nível de produtividade $(p)$ e seu gênero $(g), f(w / p, g)$, sobre a sua distribuição de produtividade dado seu gênero $F(p / g)$, ou seja:

$$
\begin{aligned}
& f_{m}(w)=\int_{p \in \Omega_{p}} d F\left(w, p / g_{w, p}=m\right)=\int_{p \in \Omega_{p}} f\left(w / p, g_{w}=m\right) d F\left(p / g_{p}=m\right) \\
& \int f\left(w, g_{w}=m, g_{p}=m\right)
\end{aligned}
$$

Sendo:

$\Omega_{p}$ : é o domínio de definição dos atributos produtivos;

$g_{p}=m:$ representam as mulheres que possuem o nível de produtividade $p$;

$g_{w}=m$ : representam as mulheres que possuem o rendimento $w$.

Supondo que a estrutura de rendimentos das mulheres, representada pela densidade condicional $f\left(w / p, g_{w, p}=m\right)$, não dependa da distribuição de produtividade dos homens, $F\left(p / g_{p}=h\right)$, a densidade contrafactual de rendimentos das mulheres, caso elas tenham o mesmo nível de produtividade dos homens, poderá ser escrita como:

$$
\begin{aligned}
& f_{m}\left(w, g_{w}=m, g_{p}=h\right)=\int_{p \in \Omega_{p}} f\left(w / p, g_{w}=m\right) d F\left(p / g_{p}=h\right) \int \\
& f_{m}\left(w / g_{w}=m, g_{p}=h\right)=\int_{p \in \Omega_{p}} f\left(w / p, g_{w}=m\right) \Psi_{p}(p) d F\left(p / g_{p}=m\right) \int
\end{aligned}
$$

Como $\Psi_{p}(p) \int d F\left(p, g_{p}=h\right) / d F\left(p / g_{p}=m\right)$, pode-se aplicar a regra de Bayes a essa equação e reescrevê-la como $\Psi_{p}(p)=\left[P\left(g_{p}=m\right) / P\left(g_{p}=h\right)\right][P$ $\left.\left(g_{p}=h / p\right) / P\left(g_{p}=m / p\right)\right]$. A probabilidade não condicional $P\left(g_{p}=m\right)$ é igual ao número de mulheres dividido pelo total de observações. A probabilidade não condicional $P\left(g_{p}=h\right)$ é definida similarmente. Como $\Psi_{p}(p)$ assume um 
número limitado de valores, pode-se estimá-la mediante um simples modelo de contagem célula por célula.

Observa-se que a equação (4) é idêntica à (5), exceto pelo fator de ponderação $\Psi_{p}(p)$. Portanto, conhecendo-se a estimativa, $\hat{\Psi}_{p}(p)$ de $\Psi_{p}(p)$, podese então estimar a equação (5) pelo método do núcleo ponderado, conforme a seguinte expressão:

$$
\hat{f}\left(w ; g_{w}=f, g_{p}=f\right)=\frac{1}{n h} \sum_{i=1}^{n} \hat{\psi}(p) K\left(\frac{w-W_{i}}{h}\right)
$$

A equação (6) é estimada da mesma forma que a equação (1), com a diferença de que (6) é ponderada pelo fator $\hat{\Psi}_{p}(p)$, o que torna esse estimador semiparamétrico.

\section{RESULTADOS}

Em todas as ocupações, o exercício contrafactual aumentou a distância entre as densidades de homens e mulheres, sendo sua distância maior nos intervalos de rendimentos mais elevados, o que indica que, embora as mulheres sejam mais produtivas, estão auferindo rendimentos menores. Os resultados, portanto, sugerem que existe uma discriminação ${ }^{3}$ contra mulheres intra-ocupação no interior de cada grupo racial, e que esta é maior para intervalos superiores da distribuição (tabela 5). A análise das densidades

Tabela 5: Razão ${ }^{4}$ do rendimento-hora contrafactual das mulheres pelo rendimento-hora dos homens nos quantis 0,25 , mediano e 0,75

\begin{tabular}{|c|c|c|c|c|c|c|}
\hline \multirow[b]{2}{*}{ Grupos Ocupacionais } & \multicolumn{3}{|c|}{ Negros } & \multicolumn{3}{|c|}{ Brancos } \\
\hline & 0,25 & Mediano & 0,75 & 0,25 & Mediano & 0,75 \\
\hline Dirigentes em geral & 0,86 & 0,66 & 0,55 & 0,72 & 0,60 & 0,59 \\
\hline Profissionais das ciências e das artes & 0,79 & 0,66 & 0,50 & 0,69 & 0,59 & 0,55 \\
\hline Técnicos de nível médio & 0,80 & 0,70 & 0,56 & 0,75 & 0,68 & 0,62 \\
\hline Trabalhadores administrativos & 0,87 & 0,79 & 0,74 & 0,77 & 0,74 & 0,75 \\
\hline Trabalhadores dos serviços & 0,77 & 0,74 & 0,75 & 0,74 & 0,71 & 0,72 \\
\hline Trabalhadores do comércio & 0,80 & 0,74 & 0,65 & 0,86 & 0,76 & 0,77 \\
\hline Trabalhadores agrícolas & 0,88 & 0,91 & 0,85 & 0,76 & 0,88 & 0,83 \\
\hline $\begin{array}{l}\text { Trab. produção de bens e serviços } \\
\text { e manutenção }\end{array}$ & 0,77 & 0,68 & 0,62 & 0,84 & 0,70 & 0,62 \\
\hline
\end{tabular}

Fonte: Elaboração própria a partir dos dados da PNAD 2004. 
também mostrou que os indivíduos de raça branca possuem uma distribuição de rendimento mais dispersa e, portanto, mais desigual, enquanto para os indivíduos da raça negra a distribuição de rendimentos é mais concentrada em torno do rendimento modal, e menos desigual. Observa-se ainda que a desigualdade de rendimentos entre gêneros é mais elevada dentro da raça branca.

As densidades de homens e mulheres gerentes estão bastante próximas; no entanto, após estas terem recebido as características produtivas dos homens elas se distanciaram, sendo o efeito do exercício contrafactual visual-

Figura 1a: Densidade do logaritmo do rendimento-hora dos gerentes/raça branca

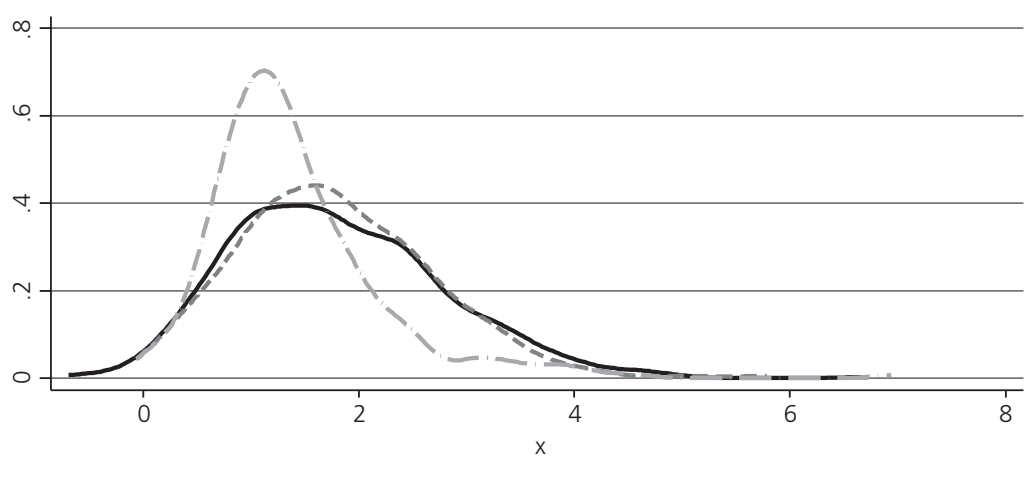

kdensit Homem

- - - - kdensit Mulher

. kdensit Simulação

Figura 1b: Densidade do logaritmo do rendimento-hora dos gerentes/raça negra

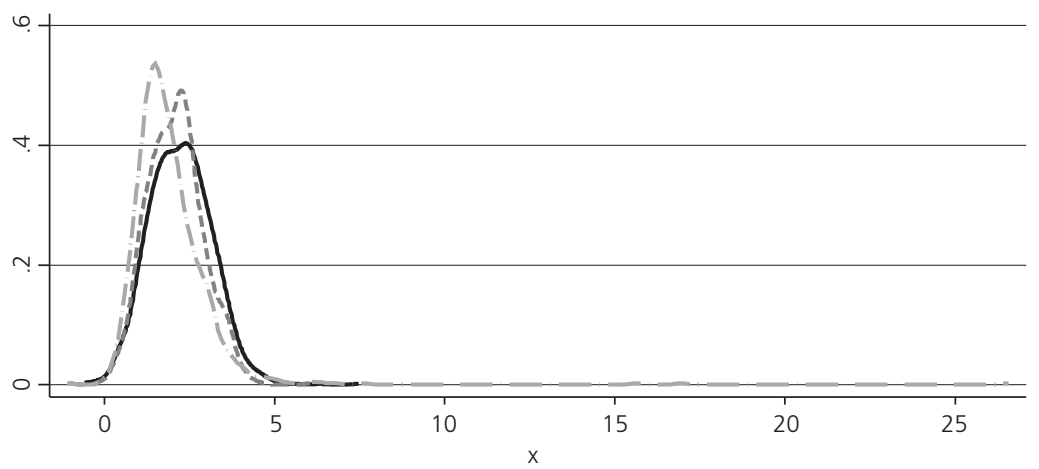

kdensit Homem 
mente mais claro para a raça branca (figuras 1a e 1b). Na tabela 5, observase uma tendência de queda na razão entre o rendimento contrafactual das mulheres e o rendimento dos homens, ou seja, a desigualdade é maior para rendimentos mais elevados.

Para o segundo grupo ocupacional, os profissionais das ciências e das artes, as densidades apresentadas nas figuras $2 \mathrm{a}$ e $2 \mathrm{~b}$ mostram a diferença visual entre a densidade de homens e mulheres. $\mathrm{O}$ fato de a densidade contrafactual das mulheres não se distanciar de forma clara da densidade original pode ser justificado pela baixa diferença nos atributos produtivos entre homens e mulheres dessa ocupação.

Figura 2a: Densidade do logaritmo do rendimento-hora

dos profissionais das ciências e das artes/raça branca

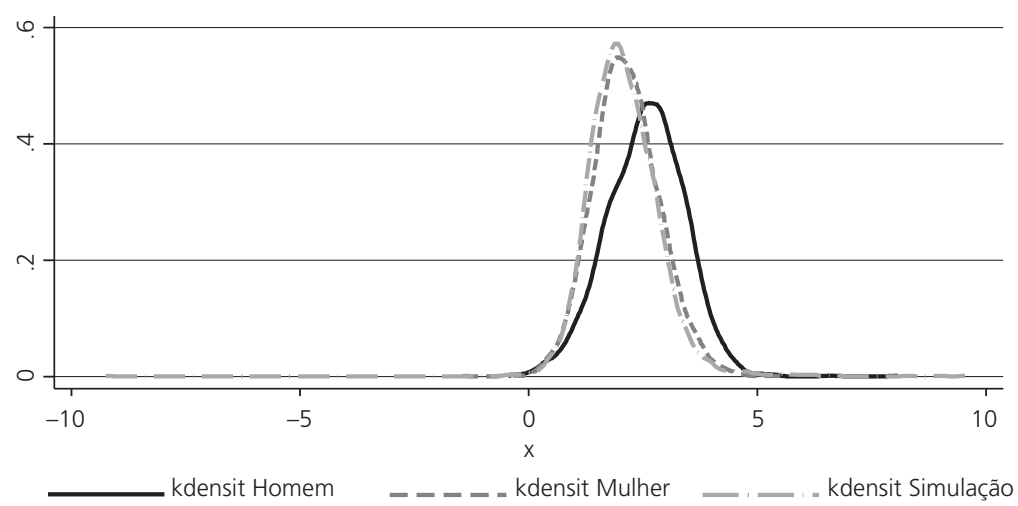

Figura 2b: Densidade do logaritmo do rendimento-hora dos profissionais das ciências e das artes/raça negra

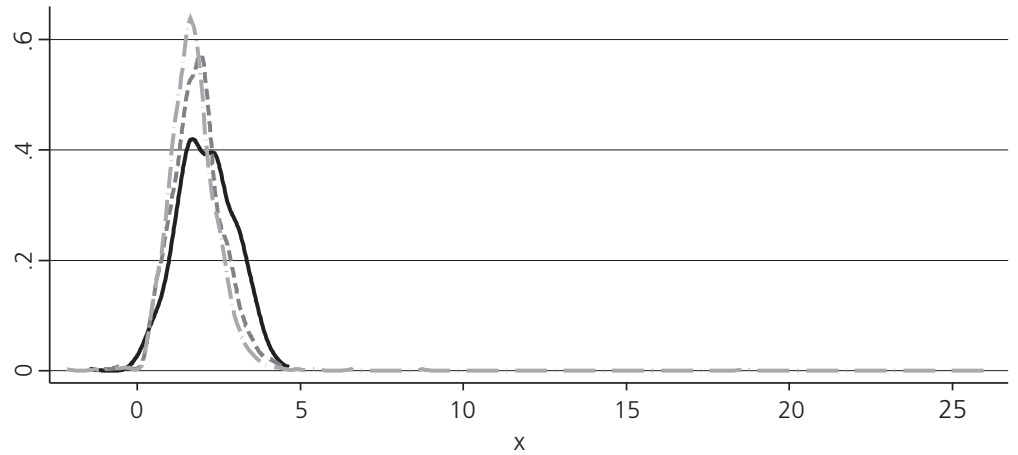

kdensit Homem 
Nas ocupações de técnicos também ocorreu uma transladação à esquerda das densidades das mulheres, após se atribuir a estas o mesmo nível de produtividade dos homens (figuras 3a e 3b). A despeito de essa ocupação concentrar, em sua maioria, profissionais com mesma escolaridade, nível médio completo, existe uma diferença considerável entre as densidades contrafactuais das mulheres e as densidades dos homens em ambas as raças. $\mathrm{O}$ aumento da desigualdade para maiores rendimentos e o menor rendimento modal na distribuição das mulheres sugerem evidências de um elevado grau de discriminação por gênero nessa ocupação (figuras 3a e $3 \mathrm{~b}$ e tabela 5).

Figura 3a: Densidade do logaritmo do rendimento-hora dos técnicos de nível médio/raça branca

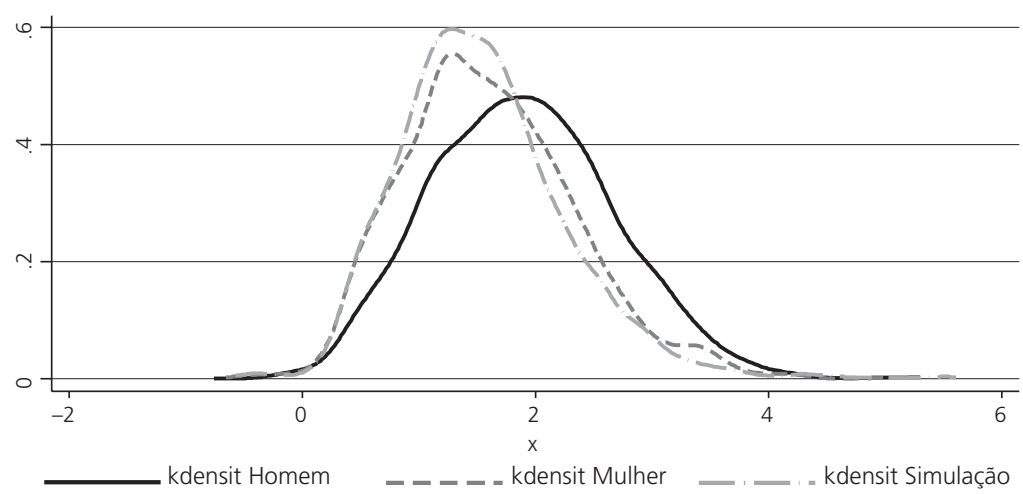

Figura 3b: Densidade do logaritmo do rendimento-hora dos técnicos de nível médio/raça negra

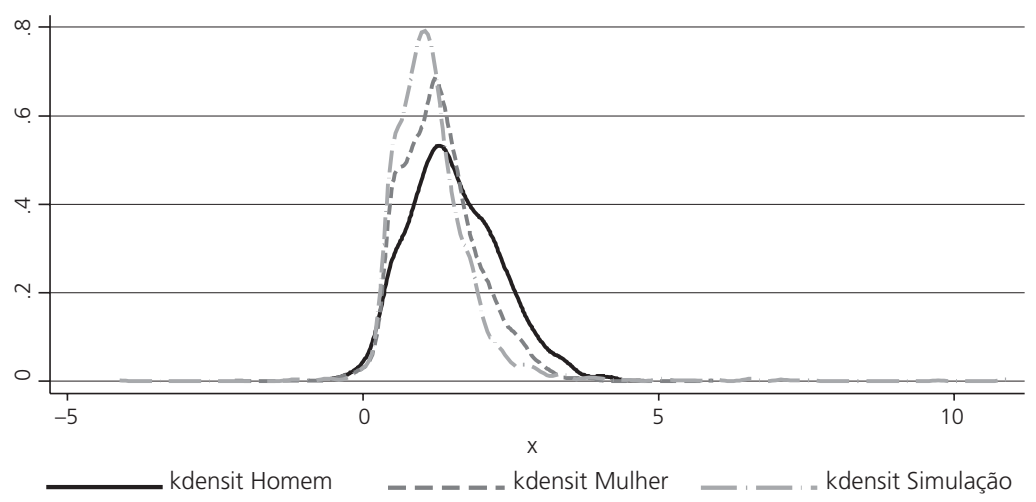


No setor administrativo, percebe-se uma baixa desigualdade entre os gêneros e uma certa semelhança entre as densidades das raças brancas e negras (figuras $4 \mathrm{a}$ e $4 \mathrm{~b}$ ). Isso ocorre provavelmente em virtude da presença do setor público, que tem dificuldades em praticar discriminação. Apesar de esse efeito positivo do setor público, na tabela 5 ainda se pode identificar uma tendência crescente na desigualdade para os quantis mais elevados da distribuição.

Para as mulheres do serviço (figuras 5 a e $5 b$ ), o exercício deixou as densidades das mulheres praticamente sobrepostas, ou seja, nesse setor, o nível

Figura 4a: Densidade do logaritmo do rendimento-hora dos trabalhadores administrativos/raça branca

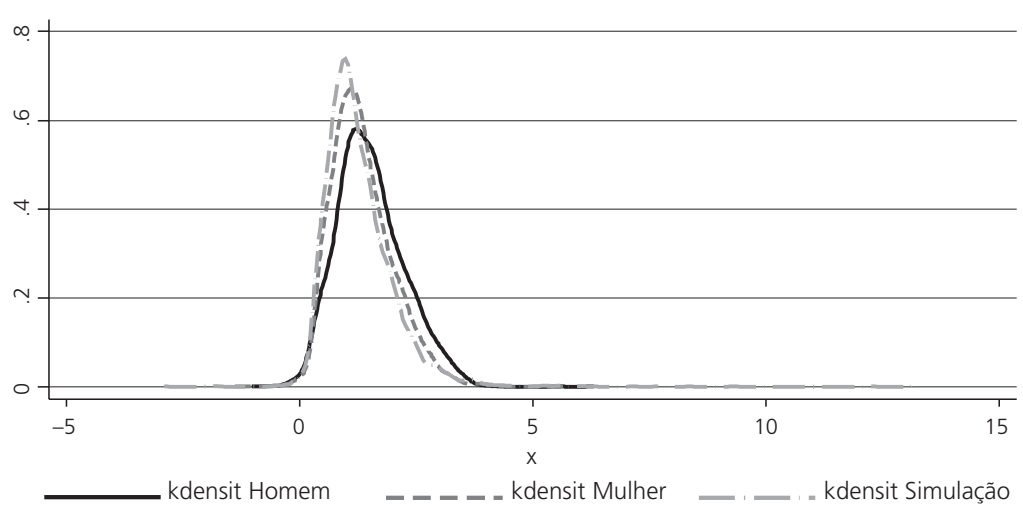

Figura 4b: Densidade do logaritmo do rendimento-hora dos trabalhadores administrativos/raça negra

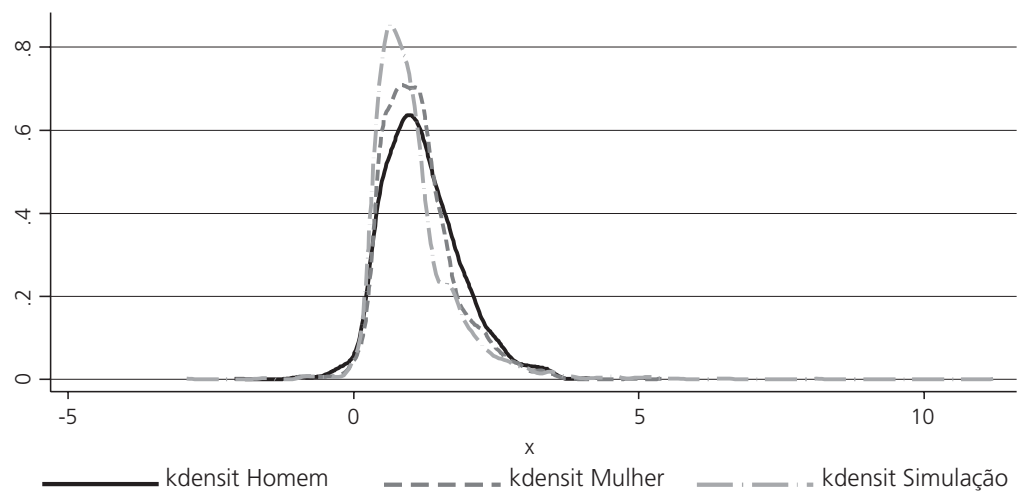


Figura 5a: Densidade do logaritmo do rendimento-hora dos trabalhadores dos serviços/raça branca

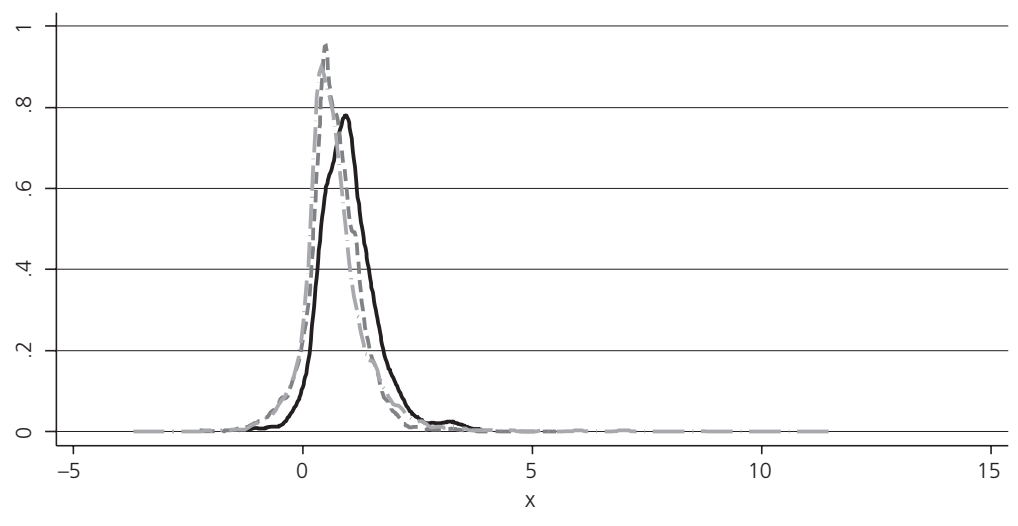

kdensit Homem

- - - - kdensit Mulher _ _ - : = kdensit Simulação

Figura 5b: Densidade do logaritmo do rendimento-hora dos trabalhadores dos serviços/raça negra

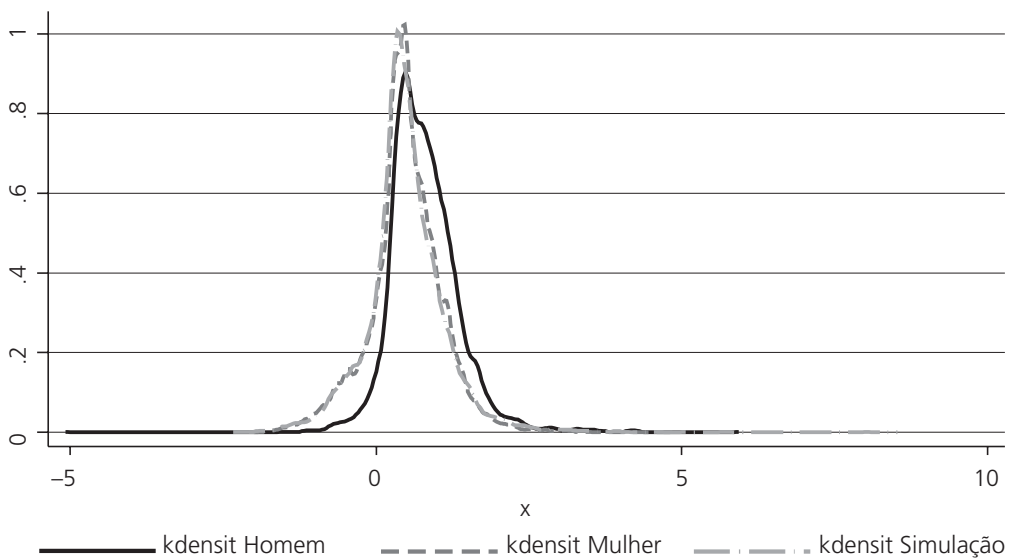

de produtividade entre homens e mulheres é praticamente o mesmo. Isso sugere que a diferença visual entre as densidades de mulheres e homens não decorre de fatores produtivos, mas sim da discriminação no interior do grupo ocupacional. A tabela 5 mostra uma maior desigualdade para trabalhadoras com rendimentos maiores e da raça branca.

No caso dos trabalhadores do comércio, houve um deslocamento da cauda superior da densidade de rendimentos das mulheres para o rendimento 
Figura 6a: Densidade do logaritmo do rendimento-hora dos vendedores do comércio/raça branca

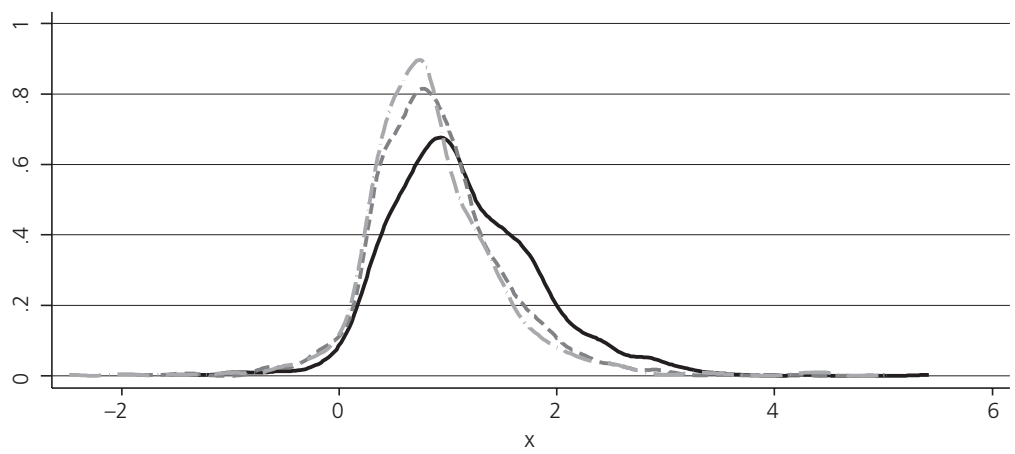

kdensit Homem

Figura 6b: Densidade do logaritmo do rendimento-hora dos vendedores do comércio/raça negra

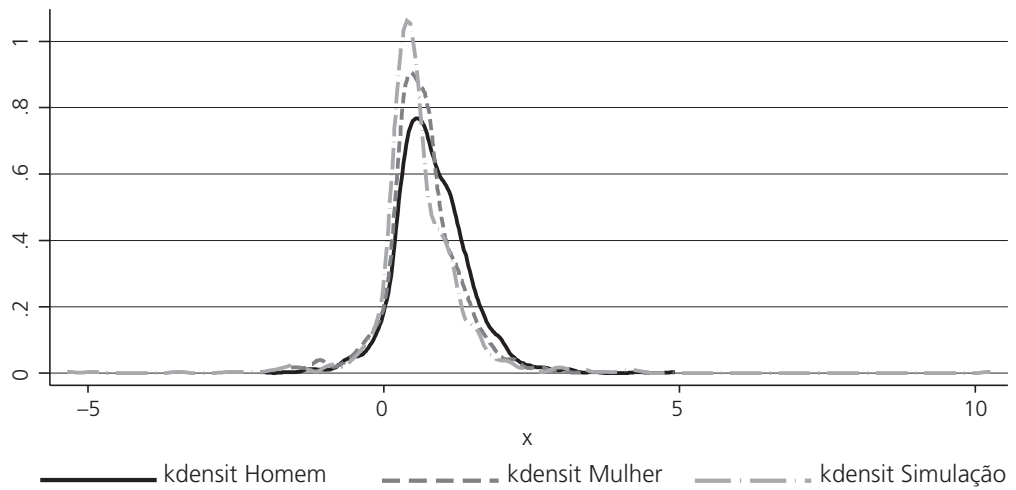

Figura 7a: Densidade do logaritmo do rendimento-hora dos trabalhadores agrícolas/raça branca

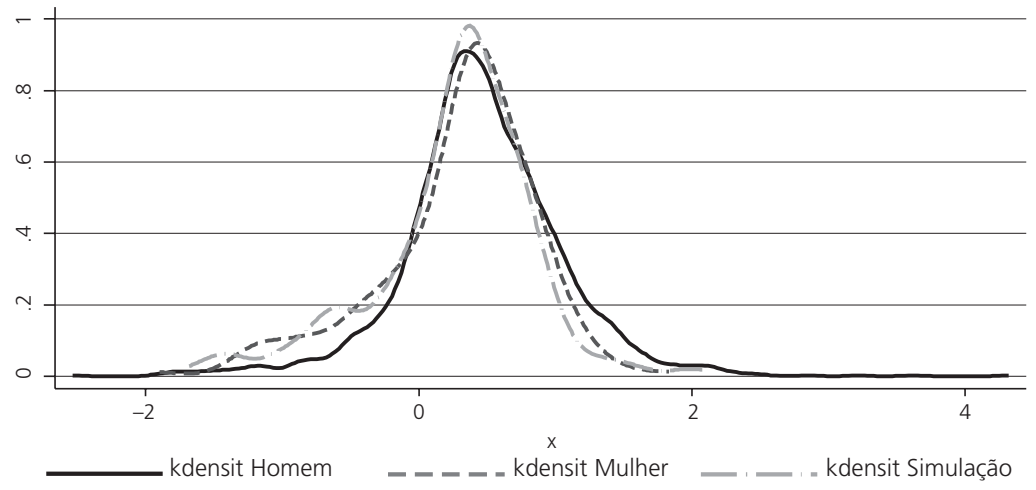


Figura 7b: Densidade do logaritmo do rendimento-hora dos trabalhadores agrícolas/raça negra

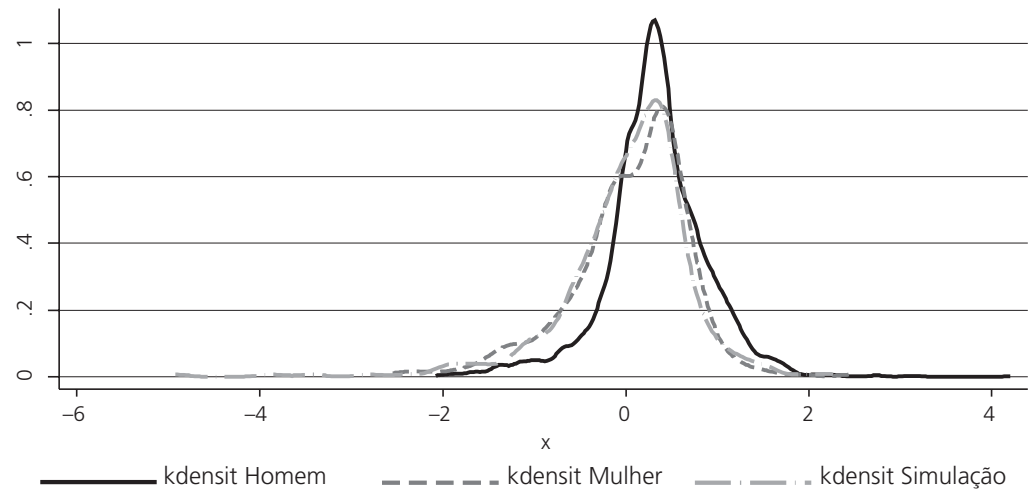

Figura 8a: Densidade do logaritmo do rendimento-hora dos trabalhadores da produção/raça branca

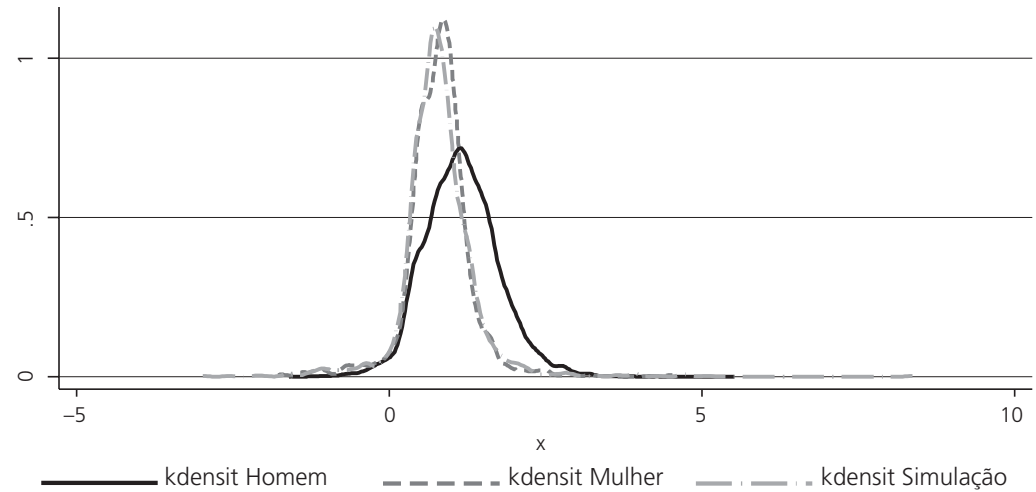

Figura 8b: Densidade do logaritmo do rendimento-hora dos trabalhadores da produção/raça negra

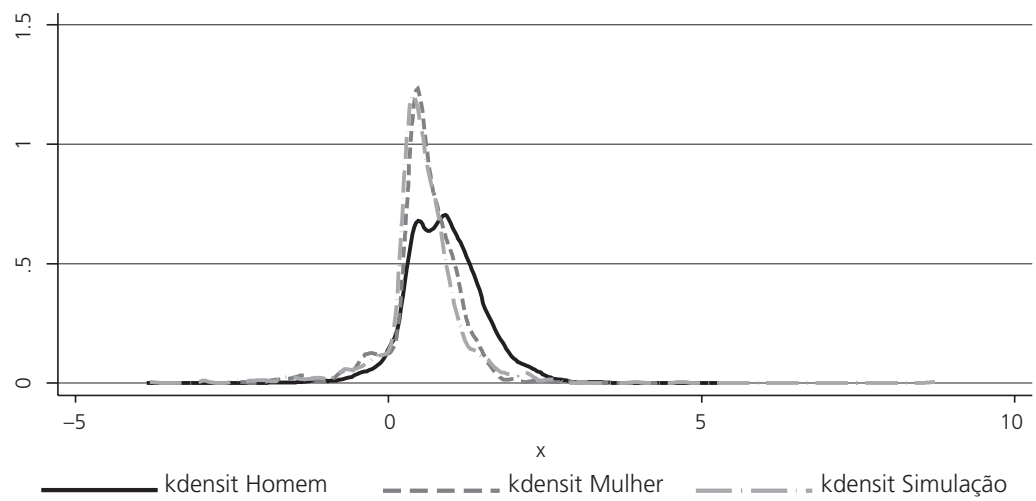


modal da distribuição, após estas receberem o nível de produtividade dos homens. A ocorrência desse deslocamento mostra que as mulheres possuem maior produtividade. Além disso, verificou-se, visualmente e mediante a análise da razão de rendimentos nos quantis (figuras 6a e 6b e tabela 5), que a desigualdade é maior para os indivíduos pertencentes à raça negra.

$\mathrm{Na}$ ocupação agrícola, os resultados das estimações diferem entre as raças. Na raça branca, a densidade contrafactual das mulheres e a densidade dos homens estão praticamente sobrepostas, existindo apenas pequena diferença entre elas no extremo direito da distribuição; portanto, não se consegue visualizar discriminação. Já para os indivíduos negros alocados nesse setor percebe-se claramente uma diferença em suas distribuições, permitindo identificar uma desigualdade de rendimentos entre gêneros.

No setor de produção de bens e serviços, no qual é dominante a presença masculina, percebe-se claramente a desigualdade entre gêneros em ambas as raças, com a distribuição das mulheres se concentrado em torno do rendimento modal, enquanto a distribuição dos homens está localizada mais à direita, ou seja, onde os rendimentos são maiores.

\section{CONCLUSÃO}

Os resultados encontrados neste estudo corroboram anteriores, ao encontrar evidências de discriminação contra mulher intra-ocupações. No entanto, algumas contribuições adicionais podem ser feitas. A desigualdade de rendimentos por gênero se mostrou mais elevada para as ocupações em que a escolaridade é maior e com tendência crescente ao longo da distribuição. Este último fato sugere que o mercado de trabalho brasileiro pode estar impedindo mulheres de ocuparem cargos de melhor remuneração e, conseqüentemente, dificultando a mobilidade social feminina e contribuindo para o grau de feminização da pobreza. Portanto, conforme estes resultados, podem-se sugerir políticas públicas de ação afirmativa, as quais promovam a diversidade no ambiente de trabalho e estimulem a participação de mulheres em cargos executivos e de chefia.

Apesar da relevância destas conclusões, vale ressaltar que nem todo o diferencial de rendimentos entre homens e mulheres decorre da discriminação, pois uma parcela deste pode ser relacionado a fatores não controlados 
nos exercícios contrafactuais. Além disso, dentro dos grupos ocupacionais considerados existem categorias extremamente amplas, o que poderia camuflar a existência de segregação ocupacional dentro do próprio grupo, e, portanto, superestimariam a discriminação salarial pura.

\section{NOTAS}

1. Considerou-se como negro o indivíduo da raça negra ou parda.

2. O índice utilizado na tabela 4 foi calculado utilizando-se a fórmula $M H_{j}=\frac{M_{j}}{H_{j}}$, em que $M_{j}$ é o número de mulheres ocupadas no grupo $j$ e $H_{j}$ é o número de homens ocupados no mesmo grupo. Esse índice foi adaptado de Rios-Neto e Baptista (1998). Se os grupos ocupacionais são predominantemente preenchidos por mulheres, índice maior do que 1; por homens, índice menor do que 1; ou integrados, índice próximo a 1.

3. Com base no conceito de discriminação e na metodologia adotada, a discriminação por gênero intragrupos ocupacionais é tida como a distância entre a densidade contrafactual das mulheres e a densidade dos homens.

4. Essa razão mostra o rendimento contrafactual das mulheres como proporção dos rendimentos dos homens, ao longo dos quantis da distribuição de rendimentos. Ela busca evidenciar uma tendência de crescimento da discriminação à medida que as mulheres se situam em faixas de rendimentos mais elevados.

\section{REFERÊNCIAS BIBLIOGRÁFICAS}

ARAÚJO, V. F., RIBEIRO, E. P. (2002) “Diferenciais de rendimentos por gênero no Brasil: uma análise regional”. In: Revista Econômica do Nordeste, v. 33, n. 2, p. 196-217.

BAPTISTA, D. (2000) "Diferenciais de rendimento e discriminação por sexo no mercado de trabalho brasileiro na década de 1990". In: Anais do XII Encontro Nacional de Estudos populacionais da ABEP, Caxambu.

BARROS, R. P., MACHADO, A. F., MEDONÇA, R. S. P. DE (1997). A desigualdade da pobreza: estratégias ocupacionais e diferenciais por gênero. Rio de Janeiro: IPEA, 1997. 36 p. Texto para discussão n. 769.

CAVALIERI, C. H., FERNANDES, R. (1998) “Diferenciais de rendimentos por gênero e cor: uma comparação entre as regiões metropolitanas brasileiras". In: Revista de Economia Política, v. 18, n. 1, p. 158-175, jan/mar.

DINARDO, J., FORTIN, N., LEMIEUX, T. (1996) "Labor market institutions and the distribution of wages, 1973-1992: a semiparametric approach”. In: Econometrica, v. 64, n. 5, p. 1.0011.044 . 
GIUBERTI, A. C., MENEZES-FILHO, N. (2005) "Discriminação de rendimentos por gênero: uma comparação entre o Brasil e os Estados Unidos". In: Revista de Economia Aplicada, v. 9, n. 3, p. 369-384, jul. set.

LAM, D., LEVISON, D. (1990) "Idade, experiência, escolaridade e diferenciais de salários: Estados Unidos e Brasil”. In: Pesquisa e Planejamento Econômico, v. 20, n. 2.

LEME, M. C. DA S., WAJNMAN, S. (2000) "Tendências de Coorte nos diferenciais de rendimento por sexo". In: R. Henriques (org.). Desigualdade e pobreza no Brasil. Rio de Janeiro: IPEA, 2000, p. 251-270.

OAXACA, R. (1973) "Male-female wage differentials in urban labor markets". In: International Economic Review, v. 14, n. 3, p. 693-709, oct.

OIT. (2003) "Relatório global sobre discriminação no trabalho: a hora da igualdade no trabalho". Genebra: OIT.

PARZEN, E. (1962) "On estimation of a probability density function and mode". In: The Annals of Mathematical Statistcs, v. 3, n. 3, 1.065-1.076, sep.

RAMOS, L., VIEIRA, M. L. (2001) Desigualdades de rendimentos no Brasil nas décadas de 1980 e 1990: evolução e principais determinantes. Rio de Janeiro: IPEA, 2001, 16 p. Texto para discussão n. 803.

RIOS-NETO, E. L. G., BAPTISTA, A. D. (1998) “Segregação ocupacional entre solteiras e casadas: o possível impacto da licença maternidade”. In: XI Encontro Nacional de Estudos Populacionais, Caxambu.

ROSENBLATT, M. (1956) "Remarks on some nonparametric estimates of a density function". In: The Annals of Mathematical Statistcs, v. 27, n. 3, p. 832-837, sep.

SILVERMAN, B. W. (1986) Density estimation for statistics and data analysis. Londres; Nova York: Chapman and Hall.

SOARES, S. S. D. (2000) O perfil da discriminação no mercado de trabalho: homens negros, mulheres brancas e mulheres negras. Brasília: IPEA, 2000. 25 p. Texto para discussão n. 769 . 